\section{El consentimiento presunto y la reciprocidad como mecanismos para aumentar la donación de órganos}

\author{
ALEJANDRA ZÚÑIGA F. ${ }^{a}$
}

\section{Presumed consent and reciprocity as means to increase organ availability for transplantation}

Chile became the third country in the world (after Israel and Singapore) to introduce the rule of reciprocity to receive an allograft. This means that if an individual opts for not being an organ donor, loses priority to receive a transplant. Despite the difficulties associated with its implementation, the recent Chilean reform that also incorporated the presumed consent, should be studied by those countries that are trying to overcome the severe lack of organ availability for transplantation.

(Rev Med Chile 2015; 143: 1331-1336)

Key words: Allografts; Presumed consent; Transplants.
Universidad de Valparaíso, Chile.

${ }^{a}$ Doctora en derecho.

Trabajo financiado por Proyecto Fondecyt Regular № 1150013 "Adjudicación de recursos sanitarios escasos, priorización y transparencia: los problema de la normativa sobre trasplante de órganos en Chile".

Recibido el 21 de mayo de 2015 , aceptado el 9 de julio de 2015.

Correspondencia a:

Alejandra Zúñiga Fajuri Universidad de Valparaíso, Chile. Errázuriz 2120 - Valparaíso, Chile. Tel.: 56-32- 2507014 /

56- 32- 2507048

alejandra.zuniga@uv.cl
E n los últimos años, la opinión técnica, política y pública en muchos países se ha inclinado a favor de la regla del "consentimiento presunto" en materia de donación de órganos para trasplante como mecanismo que mejora y promueve eficazmente la donación. En un intento por abordar los decepcionantes resultados que tuvo la reforma a la Ley de donante de órganos, Chile modificó la normativa en octubre de 2013 introduciendo importantes enmiendas que, más allá de las dificultades prácticas que revisaremos, han permitido incorporar las reglas del "consentimiento presunto" y el principio de "reciprocidad".

Este artículo revisa la experiencia chilena y las razones que le llevaron a unirse a Singapur ${ }^{1} \mathrm{e}$ Israel $^{2}$ como países pioneros en la introducción de la reciprocidad en la asignación de órganos para trasplante a fin de aumentar el registro de donantes. Se trata de una iniciativa que, sorprendentemente, ha recibido muy poca atención, a pesar de establecer un precedente notable que, según evidencia la experiencia comparada, podría convertirse en un recurso verdaderamente útil.

\section{La reforma legislativa}

Las normativas sobre trasplante de órganos pueden clasificarse en función del carácter, la condición y el ejercicio del consentimiento del donante. De este modo, es posible identificar distintos modelos de trasplante en atención, primero, a si es necesario o no que conste la voluntad expresa del donante del órgano. Si lo es, se trata de un modelo de donación "explícita" o "opting in"; de lo contrario, si se asume que todas las personas son potenciales donantes a menos que señalen lo contrario, estamos ante un modelo de "consentimiento presunto" u "opting out". Si no existe condición para ser beneficiario de una donación de órganos, estamos ante el modelo de donación "universal". Si, en cambio, la voluntad de donar se convierte en un requisito para ser receptor de un órgano (es decir, si a los individuos nos es exigible la reciprocidad), el modelo será "condicionado"3. Este es uno de los cambios que han sido introducidos por la nueva ley chilena y que sólo existía, desde 1986, en Singapur y, desde 2010, en Israel.

Las leyes de consentimiento presunto son 
desde hace muchos años, una de las principales herramientas de los países que muestran las más altas tasas de donación en el mundo. Desde ya, a España -a la cabeza en donación mundial de órganos- le siguen Bélgica, Austria, Francia, Finlandia, Polonia, Suecia, Noruega, Italia, Luxemburgo, Hungría, Grecia, República Checa y Eslovenia, entre otros. "La evidencia sugiere que el consentimiento presunto se asocia con un incremento en la tasa de donación de órganos, aun cuando haya otros factores que también influyan"4.

Si bien la relación entre la presunción de consentimiento y las tasas de donación es compleja -pues inciden también otros factores, como la disponibilidad de donantes potenciales, la infraestructura para trasplante y la inversión en el cuidado de la salud ${ }^{5}$ - los estudios realizados muestran que las leyes de consentimiento presunto tienen un índice superior de donaciones, de aproximadamente 25 a 30\%, en comparación con los países de consentimiento explícito ${ }^{6}$. Es por ello que la inmensa mayoría de los modelos de trasplante con buenos resultados son de consentimiento presunto, universal y restringido. España -junto con Austria- posee un modelo de consentimiento presunto, universal y absoluto ${ }^{7}$.

Singapur aprobó la Ley de Trasplante de Órganos Humanos (HOTA) en 1987, que aplica la regla de prioridad con un sistema opt-out. Si una persona no se opone a donar sus órganos después de la muerte, se le da automáticamente prioridad para recibir un órgano en caso de necesitarlo en el futuro ${ }^{8}$. De ese modo, el sistema proporciona un doble incentivo para la donación: evita el costo de hacerse no donante y da prioridad en la lista de espera ${ }^{9}$. Si bien la combinación de la presunción de consentimiento y priorización parece haber tenido cierto éxito en el aumento de las donaciones en Singapur ${ }^{10}$ lo cierto es que ni aún este método puede prevenir el problema de los free-riders esto es, de aquellos que se aprovechan del sistema sin aportar en nada a su mantenimiento ${ }^{11}$.

A su turno, en enero de 2010, la Organ Transplant Act 2008, que regula la donación y adjudicación de órganos, entró en vigencia en Israel estableciendo un sistema de puntos que da carácter preferencial para recibir un órgano a quienes están dispuestos a ser donantes. Como resultado el sistema establece una tabla escalonada que incluye la máxima prioridad, prioridad regular y segunda prioridad. La máxima prioridad se otorga a: (a) quienes han firmado el consentimiento de donación o a los familiares directos de personas que han donado sus órganos (b) y a quienes han donado en vida un riñón o hígado a un receptor no especificado. La prioridad regular la tienen aquellos candidatos que poseen una tarjeta de donantes. Por último, la segunda prioridad se da a quienes, sin poseer la tarjeta de donante, tienen un familiar directo que sí ha manifestado su intención de donar al momento de su muerte. "Desde la vigencia de la ley, Israel ha sido testigo de un número récord de firmas de la tarjeta de donante y se ha producido un aumento significativo en el número real de trasplantes"12.

\section{El camino recorrido en Chile}

Como se muestra a continuación, el crecimiento de la donación de órganos en Chile disminuyó drásticamente en el año 2007. Si bien los donantes aumentaron de 53 en 1993 a 147 en 2000, el crecimiento se detuvo y cayó de manera constante a partir de entonces. Esto llevó a las autoridades sanitarias a seguir el ejemplo de España en la introducción de la institución del consentimiento presunto $^{13}$ (Figura 1).

La Ley del Donación de órganos (Ley 20.413), vigente desde enero de 2010, introdujo el consentimiento presunto y estableció un Comité de Coordinación de Trasplantes y un Registro de no donantes. Para registrarse como no donante se permitió que las personas expresaran su voluntad en el Registro Civil al momento de la obtención o renovación de su carnet de identidad o licencia de conducir. Como consecuencia, los datos oficiales mostraron una drástica caída en la donación de órganos, las tasas de trasplante y el aumento considerable del registro de no donantes. De enero de 2010 a julio de 2012, unos 2.780 .223 chilenos optaron por ser no donantes al renovar los documentos de identidad o de conducir. En 2010 los donantes reales cayeron a un mínimo de 92 (el más bajo en 15 años). Incluso considerando el impacto negativo del terremoto de febrero de 2010 por la pérdida de instalaciones hospitalarias, la tendencia negativa se mantuvo ${ }^{14,15}$.

Un estudio mostró que más de $70 \%$ de los encuestados desconocían el alcance de la nueva ley, mientras que $16 \%$ consideraba que el sistema de donación y trasplante de órganos estaba de alguna 
manera sujeto a las fuerzas del mercado. Exhibiendo una enorme desconfianza y desinformación, $12 \%$ señaló que "sólo los ricos" tienen acceso a los órganos obtenidos, mientras que $13 \%$ temía que el personal sanitario "le dejara morir" a fin de hacerse con sus órganos ${ }^{16}$.

En un intento por hacer frente a los decepcionantes resultados, en octubre de 2013 Chile modificó la ley instaurando la exigencia de dejar constancia de la voluntad de no ser donante en un documento "especial" firmado ante notario, que pasaría a formar parte del Registro Nacional de No Donantes. La función de este Registro es doble. Primero, se deja constancia de quienes no desean ser donantes y, segundo, entrega una herramienta adicional a los médicos encargados de seleccionar a los receptores de órganos quienes deben colocar en segunda prioridad a los inscritos en el registro.

Según muestra la tabla, las enmiendas lograron aumentar la donación de órganos en $65 \%$ año tras año, revirtiendo la tendencia a la baja del período anterior. En general, desde 2010, los donantes de órganos han aumentado más de 100\% y el trasplante de órganos en más de la mitad. En el pasado año la donación de órganos alcanzó los 112 donantes efectivos (Figura 2).

Hay que tener presente que la reforma no revocó las declaraciones de voluntad hechas durante la vigencia de la primera etapa de la ley, de modo que las personas que eligieron ser no donantes entre los años 2010-2013 igualmente perderían prioridad para fines de trasplante de órganos lo que, sin duda, plantea serios problemas de justicia.
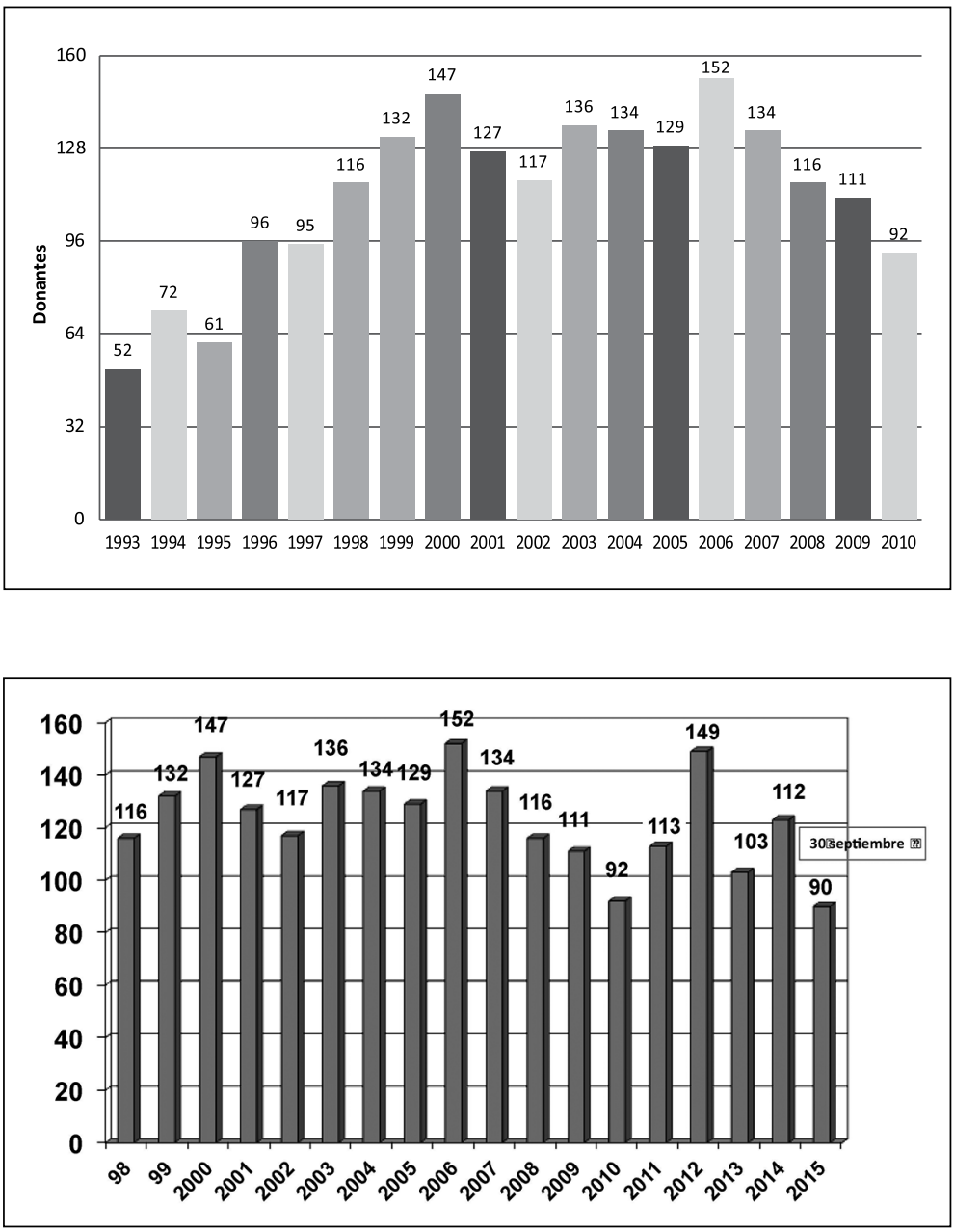

Figura 1. Donantes de órganos en Chile, 1993-2010. Fuente: Corporación del trasplante.
Figura 2. Donantes de órganos (Chile 1998-septiembre de 2014). Fuente: Ministerio de Salud. 


\section{Discusión}

La fuerte caída y el rebote subsiguiente de los índices de donación de órganos es bastante llamativa. La conclusión ineludible que se desprende de estos datos es que los chilenos son donantes sólo porque la alternativa es "demasiado complicada”. En otras palabras, la mayoría prefieren no donar. Indirectamente, esta observación significaría que los efectos positivos de un sistema de consentimiento presunto en Chile, y posiblemente en otros lugares, se basan en el hecho de que algunas personas son demasiado pasivas o cómodas como para inscribirse en el registro de no donantes. Si ese es el caso, entonces surgen preguntas sobre la legitimidad moral de la institución. En otras palabras, si es moralmente legítimo obligar a la gente a ser donante de órganos y, a su turno, penalizar a quienes no lo son despojándolos de prioridad ${ }^{17}$.

La incorporación del principio de reciprocidad en la ley pretendió transformarse en un potente incentivo para favorecer la donación de órganos. Existen razones de utilidad e instrumentales para la reciprocidad, toda vez que si se sabe que no se recibirá un órgano si no se es donante, probablemente habrá mayor voluntad para serlo y, con ello, serán más las vidas que podrán salvarse. Si una persona pretende recibir un órgano, debe estar dispuesto a su vez a dar uno propio ${ }^{18}$.

El sistema es consistente con la idea de que, ya que la oferta de donantes de órganos ha sido altamente superada por la demanda y que estamos ante lo que podemos calificar como un "recurso social muy escaso", los principios de justicia distributiva demandan la incorporación de una regla de altruismo recíproco. Ello permite corregir la injusticia derivada de los llamados "free riders", aquellas personas que utilizan recursos sociales limitados eludiendo aportar al costo de su producción. La voluntad de ser donante de órganos o tejidos a cambio de ganar la calidad de "elegible" para la recepción de los mismos, parece un requisito moral básico de justicia.

Algunos autores han derivado el deber de reciprocidad de un deber moral general llamado "deber de ayuda mutua" o "deber de socorro". Este deber moral ha sido el fundamento de las leyes promulgadas en diferentes países que obligan a todos los ciudadanos (no sólo médicos o policías) a ayudar a una persona en grave peligro, en la me- dida en que ello no signifique un costo excesivo o un riesgo real para el socorrista ${ }^{19}$.

Con el consentimiento presunto los órganos para trasplante, una vez que han sido donados, se transforman en un bien público pues es el Estado el que regula su adjudicación. A su turno, todas las personas -como ocurre con los bienes públicos- tienen derecho a ellos cuando los necesitan, incluso los free riders, pues la norma chilena establece una regla de prioridad para el trasplante, no de exclusión. Jarvis sostiene que "aquellos y sólo aquellos que eligen potencialmente contribuir al sistema, están en posición de beneficiarse de él" 20 . Ello permitiría disminuir la demanda de órganos -pues se excluiría a quienes han optado por no sumarse al esquema de donación-, aumentaría el número de donantes - pues claramente la gente preferirá formar parte del sistema $\mathrm{y}$, finalmente como beneficio marginal, se promovería la solidaridad y el altruismo en vez del egoísmo.

¿Qué críticas se han planteado a este tipo de normas? Posiblemente el cuestionamiento más importante al consentimiento presunto es la afirmación de que sí se genera un costo significativo para las personas, pues quienes no formen parte de los registros de no donantes serán más propensos a recibir una atención subóptima del personal médico cuando estén en un estado crítico, a fin de aumentar los órganos disponibles. Esta creencia es infundada pues los profesionales de la salud ven la pérdida de un paciente como un fracaso profesional y ningún médico recibe beneficios financieros por informar a las agencias de obtención de órganos que tienen un potencial donante bajo su cuidado. Por lo demás, las posibilidades de necesitar un trasplante son, de hecho, lo suficientemente bajas como para que la regla de preferencia pueda beneficiar a alguna persona en particular con una real necesidad médica ${ }^{21}$.

Otra crítica que se ha levantado en contra de la regla de prioridad dice que las personas deberían donar órganos sólo por razones altruistas y que la existencia de incentivos no altruistas envilece el carácter filantrópico del sistema. Kolber responde que los incentivos de prioridad no reducen las ocasiones para el altruismo, sino que sólo aumentan el rango de oportunidades para ser donantes potenciales. No reducen el comportamiento altruista pues los que tienen prioridad aun así están haciendo una donación, sólo que están donando a un pozo de acceso limitado ${ }^{22}$. 
Por cierto, aunque las reglas de prioridad puedan disminuir los actos altruistas, ello no significa que la reciprocidad sea una norma injusta o arbitraria. Cosa distinta, parece una regla que impide que quien toma una decisión poco solidaria pueda luego aprovecharse de quienes en su momento optaron por el altruismo. En materia de adjudicación de recursos escasos sanitarios críticos -como son los órganos para trasplantes-, si bien el criterio general es que los recursos se asignen sobre la base de la necesidad sanitaria, lo cierto es que nada impide que ese criterio se complemente con otro que promueva de mejor manera la justicia y la eficiencia, especialmente si se entiende, con Rawls, que "sólo quienes están dispuestos a hacer justicia, tienen derecho a ella"23.

Se trata, además, de una regla que promueve la eficiencia puesto que los incentivos negativos podrán servir para modificar decisiones que, muchas veces, se toman sin considerar las consecuencias para otros. Con este requisito se obliga a las personas a ser empáticas, por lo que tendrán una actitud más cuidadosa y solidaria al momento de decidir qué hacer con sus órganos ${ }^{24}$.

La directora del Servicio de Donación y Trasplante del Sistema Nacional de Salud del Reino Unido (NHS Blood and Transplant), Sally Johnson, llamó a debatir una nueva ley de trasplante que considere la inclusión del consentimiento presunto y la reciprocidad. "Almost everyone would take an organ if they needed one-but only $57 \%$ of families agreed to donation when they were asked... is it fair to take if you won't give?"25. Dicho de otra forma, es necesario considerar que, en materia de adjudicación de órganos (el recurso sanitario más escaso que existe) las consideraciones clínicas no son suficientes para tomar decisiones finales pues ¿puede ser justo tomar un órgano si no estás dispuesto a dar?

\section{Conclusiones}

La idea de ofrecer un lugar prioritario a los donantes registrados como incentivo, sumado a la regla del consentimiento presunto, permitirá que muchas más personas se hagan donantes de órganos y, con ello, se salven más vidas. Se trata de una regla moral potente que se ubica en la base de la denominada "ética Kantiana", en el sentido de actuar de tal manera que el propio comportamiento se pueda alzar en regla universal de conducta.
La vieja teoría del contrato social se sustenta en la premisa de que es posible derivar un beneficio social mayor cuando la gente actúa en su propio interés. Es decir, bajo un esquema en el que cada cual decide sobre la base de aquello que más le conviene, se actúa también en el interés de toda la comunidad promoviéndose el bien común. Además, la regla de la reciprocidad permite recibir los beneficios mientras se está vivo imponiendo los costos sólo una vez que hemos muerto lo cual, finalmente, no sólo es justo, sino que pareciera ser un buen trato.

\section{Referencias}

1. Cronin AJ. Points mean prizes: priority points, preferential status and directed organ donation in Israel. Isr J Health Policy Res 2014; 3 (1): 8. doi: http://dx.doi. org/10.1016/0379-0738(87)90048-X PMID: 3322992.

2. Iyer TK. Kidneys for transplant-“opting out” law in Singapore. Forensic Sci Int 1987; 35 (2-3): 131-40. doi: http://dx.doi.org/10.1186/2045-4015-3-8 PMID: 24565060 .

3. Glazier AK. The principles of gift law and the regulation of organ donation. Transpl Int 2011; 24 (4): 368-72. doi: http://dx.doi.org/10.1111/j.1432-2277.2011.01226.x PMID: 21276090.

4. Abadie A, Gay S. The impact of presumed consent legislation on cadaveric organ donation: a cross-country study. J Health Econ 2006; 25 (4): 599-620. doi: http:// dx.doi.org/10.1016/j.jhealeco.2006.01.003 PMID: 16490267.

5. Boyarsky BJ, Hall EC, Deshpande NA, Ros RL, Montgomery RA, Steinwachs DM, et al. Potential limitations of presumed consent legislation. Transplantation 2012; 93 (2): 136-40. doi: http://dx.doi.org/10.1097/ TP.0b013e31823173e0 PMID: 21968525.

6. Rithalia A, McDaid C, Suekarran S, Myers L, Sowden A. Impact of presumed consent for organ donation on donation rates: a systematic review. BMJ 2009; 338 jan14 2: a3162. doi: http://dx.doi.org/10.1136/bmj.a3162 PMID: 19147479.

7. Bilgel F. The impact of presumed consent laws and institutions on deceased organ donation. Eur J Health Econ 2012; 13 (1): 29-38. doi: http://dx.doi.org/10.1007/ s10198-010-0277-8 PMID: 20848298.

8. Breyer F, Kliemt H. The shortage of human organs: causes and remedies. Anal Kritik 2007; 29 (2): 188205.

9. Li D, Hawley Z, Schnier K. Increasing organ donation 
via changes in the default choice or allocation rule. J Health Econ 2013; 32 (6): 1117-29. doi: http://dx.doi. org/10.1016/j.jhealeco.2013.09.007 PMID: 24135615.

10. Chandler JA. Priority systems in the allocation of organs for transplant: should we reward those who have previously agreed to donate? Health Law J 2005; 13: 99-138. PMID: 17044324.

11. Teo B. Organs for transplantation. The Singapore experience. Hastings Cent Rep 1991; 21 (6): 10-3. doi: http:// dx.doi.org/10.2307/3562357 PMID: 1765457.

12. Datos donantes de órganos y receptores, 2014. Santiago: Ministerio de Salud; 2014. Available from: http://web. minsal.cl/sites/default/files/Resultados2014.pdf [cited 2015 Feb 2].

13. Matesanz R, Domínguez-Gil B, Coll E, de la Rosa G, Marazuela R. Spanish experience as a leading country: what kind of measures were taken? Transpl Int 2011; 24 (4): 333-43. doi: http://dx.doi.org/10.1111/j.14322277.2010.01204.x PMID: 21210863.

14. Domínguez J, Rojas JL. Presumed consent legislation failed to improve organ donation in Chile. Transplant Proc 2013; 45 (4): 1316-7. doi: http://dx.doi.org/10.1016/j. transproceed.2013.01.008 PMID: 23726561.

15. Dominguez J, Rojas JL. Presumed consent legislation failed to improve organ donation in Chile. Transplantation 2012; 94 (10S): 500. doi: http://dx.doi. org/10.1097/00007890-201211271-00957.

16. Estudio donación de óganos para trasplantes: Conocimiento y disposición de la población. Santiago: Universidad Diego Portales; 2011. Available from: http://www. udp.cl/investigacion/repo_detalle.asp?id=106 [cited 2015 Jan 23].

17. Lavee J, Ashkenazi T, Stoler A, Cohen J, Beyar R. Preliminary marked increase in the national organ donation rate in Israel following implementation of a new organ transplantation law. Am J Transplant 2013; 13 (3): 780-5. doi: http://dx.doi.org/10.1111/ajt.12001 PMID: 23279738.

18. Delmonico FL, Domínguez-Gil B, Matesanz R, Noel L. A call for government accountability to achieve national self-sufficiency in organ donation and transplantation. Lancet 2011; 378 (9800): 1414-8. doi: http://dx.doi. org/10.1016/S0140-6736(11)61486-4 PMID: 22000137.

19. Peters DA. A unified approach to organ donor recruitment, organ procurement, and distribution. J Law Health 1988-1989; 3 (2): 157-87. PMID: 11652565.

20. Jarvis R. Join the club: a modest proposal to increase availability of donor organs. J Med Ethics 1995; 21 (4): 199-204. doi: http://dx.doi.org/10.1136/jme.21.4.199 PMID: 7473637.

21. Burdick JF, Capron AM, Delmonico FL, Ravenscraft MD, Reckard CR, Shapiro M. Preferred status for organ donors: a report of the OPTN/UNOS ethics committee. Richmond: Organ Procurement \& Transplantation Network; 1993. Available from: http://optn.transplant.hrsa. gov/resources/ethics/preferred-status-for-organ-donors/ [cited 2015 Jan 23].

22. Kolber AJ. A matter of priority: transplanting organs preferentially to registered donors. Rutgers Law Rev 2003; 55 (3): 671-740. PMID: 16189910.

23. Rawls J. A theory of justice. Oxford: Oxford University Press; 1971.

24. Nolan BE, McGrath PJ. Social-cognitive influences on the willingness to donate organs. In: Shanteau J, Harris RJ, editors. Organ donation and transplantation: psychological and behavioral factors. Washington: American Psychological Association; 1990. pp. 150-8.

25. The Lancet Editorial, "Ensuring fair allocation of organs". The Lancet, Volume 382, Issue 9888, Page 181, 20 July 2013. doi:10.1016/S0140-6736(13)61582-2. 